the ices now in comets is not specified. The elongation mechanism, some versions of which have been discussed by other authors, is certainly physically plausible.

There are, of course, dangers in building theories out of such similarities: some other object not considered may have a closer resemblance; the differences may outweigh the similarities; and any single similarity may be purely coincidental. In this case, although the connection with Trojan and Hilda asteroids is intriguing, there seems to be a closer similarity between cometary nuclei and small asteroids than between cometary nuclei and the Trojan and Hilda groups of asteroids. Binzel et al. $^{3}$ have shown that the mean amplitude of brightness variation for small main-belt asteroids (average diameter $11 \mathrm{~km}$ ) is 0.33 magnitudes (about 35 per cent), and for small Earthapproaching asteroids (average diameter is $3 \mathrm{~km}$ ), after correcting for an observational basis towards larger amplitudes, the mean amplitude is 0.39 magnitudes (43 per cent). These two values are significantly larger than that for the much smaller sample of 'small' asteroids used by Hartmann and Tholen, as well as being significantly larger than the average for their sample of Trojan asteroids. The values are, in fact, the closest matches to the mean amplitude for the cometary nuclei of any groups of asteroids. This would suggest that the most appropriate connection between cometary nuclei and asteroids is through their size rather than through the site at which they formed, as small asteroids probably originated throughout the belt. The devolatilization proposed by Hartmann and Tholen would, of course, be more effective on smaller bodies which have a preponderance of surface material.

A real problem in such studies is obtaining data for a sufficiently large sample of cometary nuclei, as these objects are notoriously difficult to observe and there are strong selection effects in the observed sample. As Hartmann and Tholen note, there were only seven nuclei in their original sample (although they arbitrarily added an asteroid to their graph using the partly circular reasoning that its dynamics resemble those of a comet). The unequivocal verification that the minor planet Chiron has a cometary nature (see below) provides a datum that quite changes one's idea of the comet distribution, reinforcing the correlation with size.

Meanwhile, the approach of Hartmann and his collaborators has recently been adopted by Jewitt and $\mathrm{Luu}^{4}$ who have compared optical spectrophotometry of cometary nuclei and Trojan asteroids, much as Hartmann, Tholen and Cruikshank $\mathrm{k}^{5}$ previously compared the colours of cometary nuclei with those of asteroids identified as possible extinct comets. Using new spectrophotometric data,
Jewitt and Luu come to essentially the same conclusion, namely that the asteroids are dark and vary between slightly reddish and red and that the spectrophotometric properties of cometary nuclei fall in the same range. However, this range encompasses several different taxonomic classes of asteroids and the spectral range used to obtain the data was chosen primarily for the availability of equipment rather than for its diagnostic capability. Jewitt and Luu were limited to two cometary nuclei for which they had data, and Hartmann et al. were limited to data on three cometary nuclei, one having a much higher albedo than is typical of the dark asteroids.

The question remains, therefore, as to whether or not there really is a genetic relationship between the asteroids and the comets. There is certainly a genetic relationship in our perception of these bodies. This is exemplified by the evolving description of Chiron, which was first called 'slow moving object Kowal' in honour of its discoverer Charles Kowal, then characterized as the prototype of a new group of asteroids between Saturn and Uranus, and most recently was recognized for what it is, truly a comet exhibiting a coma of dust and (intermittently) of $\mathrm{gas}^{6-8}$. This cometary nucleus also exhibits a spectral reflectivity in the range of that of dark asteroids. An evolution of our understanding coupled with an incorrect initial classification, however, should not be mistaken for evidence of any genetic or evolutionary relationship between Chiron and asteroids. A recent analysis of the orbital evolution of Chiron by Hahn and Bailey" shows that there is a reasonably high probability that Chiron approached much closer to the Sun in the past so that its surface might be typical of highly evolved cometary nuclei such as those proposed to have evolved into Earthapproaching asteroids. Nevertheless, this can only be considered suggestive at best. It is clear that more effort is required to devise diagnostic measurements that can be made on both cometary nuclei and asteroids to show some compositional similarity. Evidence of spectral absorption features common to both would go a long way towards showing some common elements in their past evolution.

Michael F. A'Hearn is at the Astronomy Program. University of Maryland, College Park, Maryland 20742, USA.

1. Hartmann, W.K. \& Tholen, D.J. Icarus 86, 448-454 (1990)

2. Gradie, J. \& Veverka, J. Nature 283, 840-842 (1980)

3. Binzel. R. P.. Farinella, P., Zappala, V. \& Cellino, A. in Asteroids /I (eds R. P. Binze!, Gehrels, T. \& Matthews, M. S.) $416-441$ (University of Arizona Press, Tucson. 1989).

4. Jewitt. D. C. \& Luu, J. X. Astr. J. 100. 933-944 (1990)

5. Hartmann, W. K., Tholen, T. J. \& Cruikshank, P. Icarus 69 , $33-50$ (1987)

6. Meech, K. J. \& Belton, M. J. S. Astr. J. (in the press)

7. Luu, J. X. \& Jewitt, D. C. Astr. J. 100, 913-932 (1990).

8. Bus, S. J., A'Hearn, M. F., Schleicher, D. G. \& Bowell. E. L. G. Science (submitted)

9. Hahn, G. \& Bailey, M. E. Nature (in the press).

\section{Point of recall}

A SMALL barbed or asymmetric foreign object can be ratcheted through our tissues by its own ceaseless movements. Last week Daedalus devised a surgical microneedle to be manoeuvred within the body on this principle, steered by high-frequency vibro-massage pads applied to the skin. He now plans to use it on the brain itself.

Surgeons have learnt a great deal about the brain by operating on it under local anaesthetic. When searching for the causative site of an epilepsy, say, or muscular dystonia, they often find specific regions which, when touched or electrically stimulated, set off specific moods in the patient, or excite particular memories. Daedalus's micro-needle will take this process out of the operating theatre and into everyday life. Once the needle has been inserted into the brain cavity, the patient himself will be able to move it around his brain by means of a special vibro-hat. Too blunt and slow to puncture or damage the cells, the needle will nose its way harmlessly among the neurons and nerve fibres. By switching on a low-frequency magnetic field, the patient can induce currents in the needle to stimulate whatever site it has reached.

Thus the whole internal architecture of consciousness will be revealed. If a stimulated site stores a specific mood, the patient will be swept away by it; if an action, he will carry it out; if a memory - even longforgotten - it will come flooding back in full detail. If the brain really does record everything, the whole of our past life will be accessible at last. The brain may even lay down its memories in spatial diary order, so that the needle can be stepped sequentially through them. Whole sections of life could then be relived for instant autobiography or nostalgic recherche du temps perdus.

Psychiatry, of course, will be transformed. Childish memories, repressed emotions, forgotten dreams, all could be dialled up almost to order without wasting months and years on the couch. Even cure (long a dirty word among psychoanalysts, who tend to repress their inadequacies) may at last be possible. If the patient really is dogged by traumatic childhood memories, they could be tracked down by the internal needle, identified, and then erased by boosting the frequency and strength of the investigative magnetic field until induction heats the needle to cauterizing temperatures. Oedipal conflicts and penis envy could likewise be located and burnt away. Obsessive guilt, feelings of inferiority, hopeless longing, irrational fears, compulsive actions, all could neatly be cut out of patient's psyche without affecting anything else. He could even do it himself, tailoring his internal world to his own needs without surrendering his autonomy to the medical establishment.

David Jones 